\section{NEW THERAPEUTIC AVENUES IN RHEUMATOID ARTHRITIS: EXPLORING THE ROLE OF THE ADIPONECTIN-PEPITEM AXIS}

S Kemble, LE Harford, HM McGettrick*. Rheumatology Research Group, University of Birmingham, Birmingham, UK

\subsection{6/annrheumdis-2018-EWRR2018.13}

Introduction The inappropriate accumulation of leukocytes into the joint plays a significant role in the pathogenesis of rheumatoid arthritis (RA), making this process an ideal to target for therapeutic intervention. We recently identified a novel immuno-protective pathway, in which a B-cell derived peptide (PEPITEM) suppresses T-cell migration into inflamed tissues. ${ }^{1}$

Objectives Here, we examined the functionality of the adiponectin-PEPITEM axis in patients with RA and in a murine model of arthritis.

Methods Peripheral blood lymphocytes (PBL) were isolated from healthy controls or from treatment naive patients with a new onset of clinically apparent RA. PBL were treated with adiponectin or PEPITEM, and their ability to migrate across cytokine endothelial cells was assessed using phase-contrast microscopy. Mice with collagen induced arthritis (CIA) were treated with PEPITEM prior to disease onset or at the first signs of inflammation and disease incidence, severity and leukocyte infiltration were evaluated.

Results In response to adiponectin, B-cells release PEPITEM, which limits T-cell migration across inflamed endothelium in a sphingosine-1-phosphate dependent manner. This immuno-protective pathway is lost in patients with RA, where circulating B-cells express reduced levels of adiponectin receptors compared to age-matched healthy controls, and fail to respond to adiponectin and suppress T-cell migration ex vivo. This defect can be rescued with exogenous PEPITEM. Administration of synthetic PEPITEM prior to disease onset prevents CIA, significantly reducing disease incidence, clinical score, leukocyte infiltration, and bone erosion when compared to controls. PEPITEM administered at the first signs of inflammation (therapeutic intervention) in CIA also reduced clinical score, leukocyte infiltration into the inflamed joint and bone erosion when compared to controls. Unlike the S1P agonist FTY720, PEPITEM therapy did not affect leukocyte movement into and out of local draining lymph node.

Conclusions Patients with RA have a defect in adiponectin-PEPITEM axis, which potentially contributes to inappropriate accumulation of T-cells in the rheumatoid joint. Thus, re-establishing PEPITEM function to 'turn off' pathological recruitment of Tcells represents a novel and potentially powerful approach to treating patients with early rheumatoid arthritis.

\section{REFERENCE}

1. Chimen, McGettrick, et al. Nat Med 2015;21(5):467-75. doi:10.1038/nm.3842

Acknowledgements Work funded by ARUK Career Development Fellowship and Pfizer I-CRP.

Disclosure of interest S. Kemble: None declared, L. Harford: None declared, H. McGettrick Grant/research support from: Arthritis Research UK and Pfizer Research Grant

\section{PODOPLANIN (GP38), A MARKER OF SYNOVIAL INFLAMMATION, IS AN EXCELLENT THERAPEUTIC TARGET IN MOUSE COLLAGEN-INDUCED ARTHRITIS}

${ }^{1}$ GE Desanti* , ${ }^{2}$ AN Saghir, ${ }^{2}$ AJ Naylor, ${ }^{2} S$ Kemble, ${ }^{2} \mathrm{~J}$ Falconer, ${ }^{2} \mathrm{C}$ Wehmeyer, ${ }^{2} \mathrm{JL}$ Marshall, ${ }^{2} \mathrm{~K}$ Nakamura, ${ }^{3} \mathrm{M}$ Goodall, ${ }^{4} \mathrm{~L}$ Navarro-Núñez, ${ }^{4} \mathrm{SP}$ Watson, ${ }^{2} \mathrm{CD}$ Buckley. ${ }^{1}$ Institute of Microbiology and Infection; ${ }^{2}$ Institute of Inflammation and Ageing; ${ }^{3}$ Institute of Immunology and Immunotherapy; ${ }^{4}$ Institute of Cardiovascular Sciences, University of Birmingham, Birmingham, UK

\subsection{6/annrheumdis-2018-EWRR2018.14}

Introduction In patients with rheumatoid arthritis, synovial fibroblasts (SF) highly up-express the surface protein Podoplanin (PDPN) while its ligand, CLEC-2, is brought into the synovial membrane by platelets. ${ }^{1,2}$ PDPN is also up-expressed by synovial Th17 $\mathrm{T}$ cells from arthritic mice. ${ }^{3,4}$ Interestingly, IL-17 secretion by human Th17 $\mathrm{T}$ cells is triggered by direct cellular contacts with SF in a PDPN-dependent manner in vitro. ${ }^{5}$ PDPN is then an excellent biomarker and a potential regulator of joint inflammation. Despite these observations, in vivo experimental approaches that explore the therapeutic opportunity of targeting PDPN during the disease are missing. Objectives We aimed at refining the understanding of PDPN expression patterns inside the mouse synovium. We explored the therapeutic benefits from an anti-PDPN antibody in mice with auto-immune arthritis.

Methods PDPN expression patterns were investigated from freshly isolated TNFa-overexpressing mouse synoviocytes by histology and FACS. The functions of PDPN expressing synoviocytes were sought by cell sorting and quantitative PCR analysis. An anti-PDPN antibody was administrated to collagen-induced arthritis (CIA) mice from day 26 post-immunisation. The CIA mice disease activity was monitored daily until day 42 and their tissues (plasma, synovium, bones, lymph nodes) analysed by ELISA, FACS, histology, micro-CT, T cell in vitro stimulation and multiplex cytokine assays.

Results Joint inflammation triggered PDPN up-expression on a pro-inflammatory SF subset with concurrent accumulation of PDPN + anti inflammatory macrophages. These populations disappeared with the resolution of inflammation. Anti-PDPN treated CIA mice were efficiently protected from arthritis as demonstrated by their clinical features, their reduced leucocyte and non-haematopoietic cell accumulations into the joints as well as their attenuated bone erosion and remodelling. The $\mathrm{T}$ cell cytokine expression profile was normal in these mice. The anti-collagen auto-antibody plasma titres were significantly reduced in the anti-PDPN treated CIA mice compare to the control group.

Conclusions We demonstrated for the first time that PDPN is expressed by pro-inflammatory and anti-inflammatory cell subsets during joint inflammation. Moreover, we are providing strong evidences that an anti-PDPN antibody restrains autoimmune arthritis in mice. This therapeutic benefit provided by the anti-PDPN antibody correlates with a reduction of circulating auto-antibody titres. This observation suggests that the anti-PDPN antibody might interfere with the micro-environment supporting B cell activation and/or plasma cell survival. 


\section{REFERENCES}

1. Ekwall AK, et al. Arthritis Res. Ther 2011;13:R40.

2. Del Rey MJ, et al. PLoS One 2014;9:e0099607.

3. Miyamoto $Y$, et al. Molecular Immunology 2013;54:199.

4. Jones GW, et al. J. Exp. Med 2015:212:1793.

5. Noack M, et al. Arthritis Res. Ther 2016;18:148.

\section{Disclosure of interest None declared}

\section{IL-17 BLOCKADE WITH SECUKINUMAB IN PERIPHERAL SPONDYLOARTHRITIS IMPACTS SYNOVIAL IMMUNOPATHOLOGY WITHOUT COMPROMISING SYSTEMIC IMMUNE RESPONSES}

\begin{abstract}
${ }^{1} \mathrm{M}$ van de Sande*, 'L van Mens, ${ }^{2} \mathrm{~S}$ Menegatti, ${ }^{1} \mathrm{I}$ Blijdorp, ${ }^{1} \mathrm{~J}$ de Jong, ${ }^{1} \mathrm{I}$ Fluri, ${ }^{1} \mathrm{~T}$ Latuhihin, ${ }^{3} \mathrm{~A}$ van Kuijk, ${ }^{2} \mathrm{~L}$ Rogge, ${ }^{1} \mathrm{~N}$ Yeremenko, ${ }^{1} \mathrm{D}$ Baeten. ${ }^{1}$ Clinical Immunology and Rheumatology, Amsterdam Rheumatology and immunology Centre, Academic Medical Center-University of Amsterdam, Amsterdam, Netherlands; ${ }^{2}$ Immunoregulation Unit, Institut Pasteur, Paris, France; ${ }^{3}$ Amsterdam Rheumatology and immunology Centre, Reade, Amsterdam, Netherlands
\end{abstract}

\subsection{6/annrheumdis-2018-EWRR2018.15}

Introduction Secukinumab (anti-IL-17A) is an effective therapy for ankylosing spondylitis(AS) and psoriatic arthritis (PsA), the prototypical forms of spondyloarthritis (SpA).

Objectives This study assessed if secukinumab modulates the immunopathology of target lesions without blunting systemic immune responses, using peripheral $\mathrm{SpA}(\mathrm{pSpA})$ as model.

Methods 20 active peripheral SpA patients were included in a 12 week open-label trial with secukinumab (300 mg weekly for 4 weeks followed by every 4 weeks). Outcomes included clinical response, cytokine production by peripheral blood cells using TruCulture technology, and histological and qPCR analysis of synovial biopsies before and after treatment.

Results All patients completed the 12 week study, without SAEs or severe treatment-related AEs. The primary efficacy endpoint, EULAR DAS 28 response at wk 12, was achieved by $18 / 20$ patients (10 good and 8 moderate responders), with rapid and significant improvements in all clinical disease activity measurements. Clinical improvement in joint counts was associated with histological decrease in synovial sublining macrophages $(p=0.028)$ and neutrophils $(p=0.004)$, sensitive synovial biomarkers of response in $\mathrm{pSpA}$, as well as with decreased synovial expression of IL-17A $(p=0.010)$ but not TNF. Systemically, secukinumab treatment decreased CRP $(\mathrm{p}<0.01)$ and ESR $(\mathrm{p}<0.01)$, as well as MMP-3 production in the truculture system $(\mathrm{p}<0.01)$. With exception of IL-17A itself, however, the capacity of peripheral blood cells to produce a broad panel of cytokines and chemokines upon stimulation with microbial antigens was not affected.

Conclusions This mechanism-of-action study in $\mathrm{pSpA}$ indicates that clinical improvement upon secukinumab treatment is paralleled by immunomodulation of the inflamed target tissues without compromising systemic immune responses.

Disclosure of interest M. van de Sande Grant/research support from: Novartis, Eli Lilly, Boehringer Ingelheim, Consultant for: Abbvie, Novartis, Speakers bureau: x, L. van Mens: None declared, S. Menegatti: None declared, I. Blijdorp: None declared, J. de Jong: None declared, I. Fluri: None declared, T. Latuhihin: None declared, A. van Kuijk Grant/research support from: x, Consultant for: Novartis, Celgene, L. Rogge: None declared, N. Yeremenko: None declared, D. Baeten Grant/research support from: Pfizer, MSD, Abbvie, UCB, Novartis, Janssen, Boehringer Ingelheim, Consultant for:
Pfizer, MSD, Abbvie, UCB, Novartis, Janssen, Boehringer Ingelheim, Eli Lily, Roche, BMS, Glenmark, Employee of: $\mathrm{UCB}$

\section{SYNOVIAL IL-17+ CD8+ T CELLS ARE A PRO- INFLAMMATORY TISSUE RESIDENT POPULATION ENRICHED IN SPONDYLOARTHRITIS}

${ }^{1} \mathrm{KJ}$ Steel ${ }^{*},{ }^{1} \mathrm{~S}-\mathrm{Y}$ Wu, ${ }^{1} \mathrm{U}$ Srenathan, ${ }^{2} \mathrm{E}$ Chan, ${ }^{2} \mathrm{BW}$ Kirkham, ${ }^{1} \mathrm{LS}$ Taams. ${ }^{1}$ Centre for Inflammation Biology and Cancer Immunology, School of Immunology and Microbial Sciences, King's College London; 'Department of Rheumatology, Guy's and St Thomas' Hospital, London, UK

\subsection{6/annrheumdis-2018-EWRR2018.16}

Introduction Spondyloarthritis (SpA) describes a group of inflammatory joint diseases affecting $\sim 1 \%$ of the population. SpA has strong genetic associations with $H L A-B / R U N X 3$ implying a role for CD8 $+\mathrm{T}$ cells. Furthermore, genetic associations with IL23R/TRAF3IP2 and the clinical efficacy of IL-17 blockade in SpA, indicate a role for IL-17 in these diseases.

Objectives This provides a strong rationale to investigate the presence, phenotype and functional capacity of IL-17 +CD8 $+\mathrm{T}$ cells in the joints of patients with SpA.

Methods Mononuclear cells were isolated from peripheral blood (PB) and synovial fluid (SF) from patients with PsA, other peripheral-SpA types (including ankylosing spondylitis/ non-radiographic axial $\mathrm{SpA} /$ reactive arthritis/enteropathic arthritis/undifferentiated $\mathrm{SpA}$ ) and rheumatoid arthritis (RA). Cells were stimulated ex-vivo before analysis of surface marker/cytotoxic molecule/cytokine expression by flow cytometry or cytokine secretion assay (CSA). Sorting was performed on unstimulated SFMC and gene expression analysis performed by RT-PCR.

Results Frequencies of IL-17 +CD8 + T cells were increased in the SF of PsA $(p<0.0001)$ and SpA $(p=0.0009)$, but not RA patients $(p=0.3)$ vs. paired PB, with IL-17 secretion confirmed by CSA. Phenotypically, SF IL-17 +CD $8+\mathrm{T}$ cells were largely composed of TCRab + T cells $(\sim 95 \%)$, with small proportions of MAIT/NK/gd-T-cells (all <5\%). Considerable proportions of SF IL-17 + CD8 + T cells expressed markers typical of skin/gut tissue residency including $\beta 7$ integrin (median-66\%), CD49a $(57 \%)$, and cutaneous lymphocyte antigen (27\%), as well as Th17-associated markers (CCR6/CD161 expression). Interestingly, SF IL-17 + CD8 + cells expressed hallmarks of tissue resident memory $\mathrm{T}$ cells ( $\mathrm{T}_{\mathrm{RM}}$; CD45RA-CCR7-CD103+) whilst sorted CD8 $+\mathrm{CD} 69+\mathrm{CD} 103+\mathrm{T}_{\mathrm{RM}}$ cells from the PsA joint were enriched for IL-17, and expressed RORC transcript. Functionally, a high frequency of SF IL-17 + CD8+T cells coexpressed granzyme $\mathrm{B}$ and the pro-inflammatory cytokines IFN- $\gamma$, GM-CSF, TNF- $\alpha$, some IL-21 and IL-22, but very little anti-inflammatory IL-10.

Conclusions These novel findings show an enrichment of IL$17+\mathrm{CD} 8+\mathrm{T}$ cells in the joints of patients across multiple SpA types and identify a phenotypic signature for IL-17 + CD8+T cells, consisting of type 17 and tissue-associated markers. Our data demonstrate, to our best knowledge for the first time, the presence of $\mathrm{T}_{\mathrm{RM}}$ cells in the PsA joint. Functionally, IL$17+\mathrm{CD} 8+\mathrm{T}$ cells exhibit cytotoxic potential and co-express pro-inflammatory cytokines, suggesting these cells are important contributors to the pathogenesis of PsA and other SpA.

Acknowledgements This study was by supported by King's Health Partners R and D challenge award, Novartis and the National Institute for Health Research (NIHR) Biomedical 